\title{
The dynamics of the radiative envelope of rapidly rotating stars
}

\section{A spherical Boussinesq model}

\author{
M. Rieutord \\ Laboratoire d'Astrophysique de l'observatoire Midi-Pyrénées, UMR 5572, CNRS et Université Paul Sabatier, \\ 14 avenue E. Belin, 31400 Toulouse, France \\ e-mail: rieutord@ast.obs-mip.fr
}

Received 28 October 2005 / Accepted 18 January 2006

\begin{abstract}
Context. The observations of rapidly rotating stars are increasingly detailed and precise thanks to interferometry and asteroseismology; two-dimensional models taking into account the hydrodynamics of these stars are very much needed.

Aims. A model to study the dynamics of baroclinic stellar envelopes is presented.

Methods. This model treats the stellar fluid with the Boussinesq approximation and assumes that it is contained in a rigid spherical domain. The temperature field and the rotation of the system generate the baroclinic flow.

Results. We give an analytical solution to the asymptotic problem at small Ekman and Prandtl numbers. We show that, provided the Brunt-Väisälä frequency profile is smooth enough, differential rotation of a stably stratified envelope takes the form a fast rotating pole and a slow equator while it is the opposite in a convective envelope. We also show that at low Prandtl numbers and without $\mu$-barriers, the jump in viscosity at the core-envelope boundary generates a shear layer staying along the tangential cylinder of the core. Its role in mixing processes is discussed.

Conclusions. Such a model provides an interesting tool to investigate the fluid dynamics of rotating stars in particular for the study of the various instabilities affecting baroclinic flows or a dynamo effect.
\end{abstract}

Key words. hydrodynamics - stars: rotation

\section{Introduction}

Thanks to the development of new observational techniques, like interferometry or high precision photometry, rapidly rotating stars are increasingly focusing the interest of the scientific community. The best example is the nearby star Altair whose shape, rotation and inclination of axis have been determined by interferometry (de Souza et al. 2005; Peterson et al. 2006) and which has also been identified as an oscillating $\delta$-Scuti star (see Buzasi et al. 2005). Modelling such stars is therefore a challenge which needs to be taken up in order to extract the best science from these observations.

The role of rotation in the physics of stars appears at various levels. First is the determination of the shape and thermal structure which control the way we "see" the star and at this stage the differential rotation is certainly important. Then, if we are concerned by the eigenspectrum, in addition to the shape (which influences the frequency and the visibility of the modes, see Lignières et al. 2005), we also need to know the distribution of elements able to excite eigenmodes through the $\kappa$-mechanism. Finally, it has long been known that rotation drives many hydrodynamical instabilities in stably stratified radiative zones which inevitably lead to some small-scale turbulence (e.g. Spiegel \& Zahn 1970). Much work has already been done in this direction following Zahn (1992), in particular to understand the surface abundances in stars across the HR diagram (see Maeder \& Meynet 2000).

The case of rotation is therefore fundamental in stellar physics and needs to be well understood. Presently, the difficulties concentrate on the hydrodynamical effects generated by the associated inertial accelerations (Coriolis and centrifugal ones). Indeed, the analysis of these flows is quite demanding as it requires two-dimensional models.

The present paper focuses on one aspect of the dynamics of rotating stars: namely, we want to specify the shape of the baroclinic flow inside the radiative envelope of a rapidly rotating star. In such stars, it is well known that the Eddington-Sweet time scale is short enough for a steady state to be reached. But this steady flow, in which viscous effects need to be taken into account, has never been computed in its full two-dimensionality.

Previous work on this subject is quite scarce. First attempts include those of Tassoul \& Tassoul (1982, 1983a,b, 1984, 1986) who, for analytical tractability, restricted the velocity field to its first Legendre polynomial component and therefore give an almost one dimensional description of the flow. Still in the quasione-dimensional approach, the work of Zahn (1992) presents a self-consistent description based on the assumption of a strong horizontal turbulent viscosity compared to the vertical one. Later, Uryu \& Eriguchi $(1994,1995)$ touched upon the question of modeling rotating baroclinic stars in two dimensions but their neglect of viscosity removed any meridian circulation and left differential rotation incomplete. Including viscosity, Garaud (2002) investigated the dynamics of the radiative core of the sun stressed by the differential rotation of the convective zone; in this latter case the flow is essentially driven by the boundary conditions. This is certainly the most advanced work on stellar baroclinic flows to date but baroclinicity is not the main driving.

Although the full solutions are still not known, the stability of some generic situation of baroclinic shear flows has 
been investigated by Knobloch \& Spruit (1982, 1983); Knobloch (1985); Schneider (1990) and are reviewed in Zahn (1993).

Computing the baroclinic flow in a centrifugally distorted envelope with realistic density and temperature profiles is not an easy task. Therefore, we concentrate on a simpler version of such a flow, namely a "Boussinesq version in spherical geometry", which still contains a good deal of the actual physics of the problem. This model has two purposes: to reveal the main dynamical features of such flows and eventually find some robust property that may persist in actual stars and to devise a model in which hydrodynamics can be investigated more easily and which can be used as a template for the construction of more elaborated models.

The next section of the paper describes our approach including the simplifications that we use and the physics we keep. In Sect. 3, we discuss the asymptotic properties of this model in the limit of small Ekman numbers, appropriate to stellar applications. Then, after describing the numerical method, we investigate some aspects of the general case. Conclusions and outlooks follow.

\section{The model}

\subsection{Description}

We consider a system in which a self-gravitating fluid of constant density is enclosed in an undeformable sphere of radius $R$. The gravitational field is thus simply $\boldsymbol{g}=-\boldsymbol{g} \boldsymbol{r}$ where the radial coordinate is scaled with $R$ (i.e. $0 \leq r \leq 1)$ and $g$ is the surface gravity. The thermal and mechanical equilibrium of this fluid is governed by:

$$
\left\{\begin{array}{l}
-\boldsymbol{\nabla} P_{\mathrm{eq}}+\rho_{\mathrm{eq}} \boldsymbol{g}=\mathbf{0} \\
\boldsymbol{\nabla} \cdot\left(\chi \boldsymbol{\nabla} T_{\mathrm{eq}}\right)+Q=0 \\
\rho_{\mathrm{eq}}=\rho_{0}\left(1-\alpha\left(T_{\mathrm{eq}}-T_{0}\right)\right)
\end{array}\right.
$$

where $\alpha$ is the dilation coefficient, $\chi$ the thermal conductivity and $Q$ the heat sinks (since we study a stably stratified situation). The essential characteristic of this equilibrium is the Brunt-Väisälä frequency profile

$N^{2}(r)=\alpha \frac{\mathrm{d} T_{\mathrm{eq}}}{\mathrm{d} r} g(r)$

We now let this system rotate at an angular velocity $\Omega$ around the $z$-axis. In the rotating frame, because of the combination of rotation and stratification, a steady baroclinic flow appears. It is a solution of

$$
\begin{aligned}
\rho(2 \boldsymbol{\Omega} \wedge \boldsymbol{v}+\boldsymbol{v} \cdot \boldsymbol{\nabla v}) & =-\boldsymbol{\nabla} P+\rho\left(\boldsymbol{g}+\Omega^{2} \boldsymbol{e}_{s}\right)+\mu \Delta \boldsymbol{v} \\
\rho c_{p} \boldsymbol{v} \cdot \boldsymbol{\nabla} T & =\boldsymbol{\nabla} \cdot(\chi \boldsymbol{\nabla} T)+Q
\end{aligned}
$$

where $\mu$ is the dynamical shear viscosity, $c_{\mathrm{p}}$ the specific heat capacity at constant pressure, $s$ the radial cylindrical coordinate and $\boldsymbol{e}_{s}$ the associated unit vector. We decompose the thermodynamical quantities into their equilibrium and fluctuating parts, namely

$$
P=P_{\mathrm{eq}}+\delta P, \quad \rho=\rho_{\mathrm{eq}}+\delta \rho, \quad T=T_{\mathrm{eq}}+\delta T .
$$

After subtracting Eq. (1), the momentum and heat equations read:

$$
\begin{aligned}
& \rho(2 \boldsymbol{\Omega} \wedge \boldsymbol{v}+\boldsymbol{v} \cdot \boldsymbol{\nabla v})=-\boldsymbol{\nabla} \delta P+\delta \rho\left(\boldsymbol{g}+\Omega^{2} \boldsymbol{e}_{s}\right) \\
& +\rho_{\mathrm{eq}} \Omega^{2} s \boldsymbol{e}_{s}+\mu \Delta \boldsymbol{v} \\
& \rho c_{p}\left(\boldsymbol{v} \cdot \boldsymbol{\nabla} T_{\mathrm{eq}}+\boldsymbol{v} \cdot \boldsymbol{\nabla} \delta T\right)=\boldsymbol{\nabla} \cdot(\chi \boldsymbol{\nabla} \delta T) .
\end{aligned}
$$

These equations are further simplified by using the Boussinesq approximation which yields:

$$
\begin{array}{rlrl}
2 \Omega \wedge \boldsymbol{v}+\boldsymbol{v} \cdot \boldsymbol{\nabla} \boldsymbol{v} & =-\boldsymbol{\nabla} \delta P-\alpha \delta T\left(\boldsymbol{g}+\Omega^{2} s \boldsymbol{e}_{s}\right) \\
\boldsymbol{v} \cdot \boldsymbol{\nabla} T_{\mathrm{eq}}+\boldsymbol{v} \cdot \boldsymbol{\nabla} \delta T & =\kappa \Delta \delta T . & -\alpha \delta T_{\mathrm{eq}} \Omega^{2} s \boldsymbol{e}_{s}+v \Delta \boldsymbol{v}
\end{array}
$$

We removed the barotropic contribution of the centrifugal acceleration, $\rho_{0} \Omega^{2} \boldsymbol{s} \boldsymbol{e}_{s}$; fluctuations of density are retained whenever they are multiplied by an acceleration $\left(\boldsymbol{g}\right.$ or $\left.\Omega^{2} \boldsymbol{s} \boldsymbol{e}_{s}\right)$ as should be done when using the Boussinesq approximation. This approximation implies the Cowling approximation (i.e. the neglect of variations of self-gravity). We also simplified the system by assuming a constant heat conductivity. Taking the curl of the momentum equation we derive the equation of vorticity:

$$
\begin{array}{r}
\boldsymbol{\nabla} \times\left(2 \boldsymbol{\Omega} \wedge \boldsymbol{v}+\boldsymbol{v} \cdot \boldsymbol{\nabla v}+\alpha \delta T\left(\boldsymbol{g}+\Omega^{2} \boldsymbol{e}_{s}\right)-v \Delta \boldsymbol{v}\right)= \\
-\varepsilon N^{2}(r) \sin \theta \cos \theta \boldsymbol{e}_{\varphi}
\end{array}
$$

where we used the Brunt-Väisälä frequency profile (2) and introduced $\varepsilon=\Omega^{2} R / g$, the ratio of centrifugal acceleration to gravity. These equations are of course completed by the equation of mass conservation which reads

$\boldsymbol{\nabla} \cdot \boldsymbol{v}=0$

at the Boussinesq approximation.

The foregoing equations show that our problem is that of a forced flow driven by the baroclinic torque $-\varepsilon N^{2}(r) \sin \theta \cos \theta \boldsymbol{e}_{\varphi}$.

We may wonder at this stage how such a system compares with a real star and especially its baroclinicity. In a star, the isothermal and isentropic surfaces are more spherical than the equipotentials or isobars (e.g. Busse 1982); since the stellar envelope is stably stratified, entropy increases outwards which implies that, on an equipotential, entropy also increases from pole to equator. In our Boussinesq model, entropy (actually potential temperature) is represented by temperature; our equipotentials being oblate ellipsoids and the temperature gradient being positive outwards, we see that there too, entropy increases from pole to equator of an equipotential surface. As shown by Zahn (1974), the baroclinic torque is proportional to the latitudinal gradient of entropy on an equipotential.

\subsection{Scaled equations}

Gathering vorticity, energy and continuity equations, we need to solve the following system:

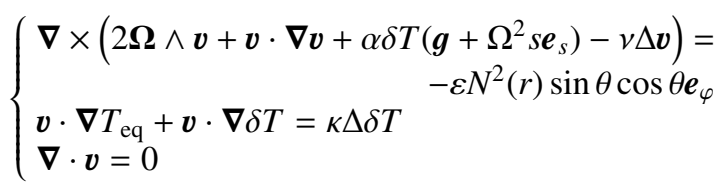

since this is a forced problem, we wish to have a forcing of order unity as well as the solution. These considerations lead us to the following scaling of the velocity field and temperature perturbations:

$\boldsymbol{v}=\frac{\Omega \mathcal{N}^{2} R^{2}}{2 g} \boldsymbol{u}, \quad \delta T=\varepsilon T_{*} \theta \quad$ with $\quad \mathcal{N}^{2}=\frac{\alpha T_{*} g}{R}$

where $\mathcal{N}$ is the scale of the Brunt-Väisälä frequency. This scaling takes into account the fact that the baroclinic flow and the associated temperature perturbations vanish when either rotation 
or the Brunt-Väisälä frequency vanishes. We thus find the system of dimensionless dependent variables:

$$
\left\{\begin{array}{l}
\boldsymbol{\nabla} \times\left(\boldsymbol{e}_{z} \wedge \boldsymbol{u}+\operatorname{Ro} \boldsymbol{u} \cdot \boldsymbol{\nabla u}-\left(r \boldsymbol{e}_{r}-\varepsilon s \boldsymbol{e}_{s}\right)-E \Delta \boldsymbol{u}\right)= \\
\left(n_{T}^{2} / r\right) u_{r}+\varepsilon \boldsymbol{u} \cdot \boldsymbol{\nabla} \theta=\tilde{E}_{T}^{2} \Delta \theta \\
\boldsymbol{\nabla} \cdot \boldsymbol{u}=0
\end{array}\right.
$$

where we introduced the numbers:

$$
E=\frac{v}{2 \Omega R^{2}}, \quad \tilde{E}_{T}=\frac{\kappa}{2 \Omega R^{2}}\left(\frac{2 \Omega}{\mathcal{N}}\right)^{2}, \quad \text { Ro }=\frac{\mathcal{N}^{2} R}{4 g}
$$

$E$ is the Ekman number which measures the viscosity, $\tilde{E}_{T}$ measures heat diffusion and Ro is the Rossby number. In addition to these numbers we will need the Froude number, Fr, the Prandtl number $\mathcal{P}$ and the $\lambda$-parameter introduced by Garaud (2002); these are respectively:

$\mathrm{Fr}=\frac{V}{\mathcal{N} R}=\frac{\Omega \mathcal{N} R}{2 g}, \quad \mathcal{P}=\frac{v}{\kappa}, \quad \lambda=\frac{E}{\tilde{E}_{T}}=\mathcal{P} \frac{\mathcal{N}^{2}}{4 \Omega^{2}}$.

$n_{T}^{2}(r)$ is the scaled Brunt-Väisälä frequency.

\subsection{Boundary conditions}

This systems needs to be completed by boundary conditions. We assume the regularity of the solutions at the sphere's centre and impose stress-free boundary conditions on the velocity field at the outer surface. Thus doing, the velocity field is determined up to a solid rotation; if $\boldsymbol{u}$ is a solution of Eq. (5) then $\boldsymbol{u}+A \boldsymbol{e}_{z} \times \boldsymbol{r}$ is also a solution ( $A$ is an arbitrary constant). For actual stars, such a degeneracy is lifted by initial conditions and conservation of angular momentum. Here, we lifted it by imposing that the solution $\boldsymbol{u}$ of Eq. (5) has no total angular momentum, i.e. that

$\int_{(V)} s u_{\varphi} \mathrm{d} V=0$

so that the total angular momentum of the star is in the background solid rotation measured by $\Omega$ (which therefore appears as the mean rotation rate).

We further complete Eq. (5) by also imposing a zero temperature fluctuation on the outer surface.

\subsection{Discussion}

As can be seen, the problem is controlled by a large number of parameters namely

- the ratio of the centrifugal acceleration $\varepsilon$ to surface gravity which is also the ellipticity of equipotentials;

- the Rossby number Ro;

- the diffusion coefficients, $E, \tilde{E}_{T}$;

- the profile of Brunt-Väisälä frequency $n_{T}^{2}(r)$.

Moreover, two other parameters will be necessary to described molecular weight gradients, namely the Brunt-Väisälä frequency profile $n_{\mu}^{2}(r)$, and the related diffusion coefficient, while another one will characterize the viscosity jump at the core-envelope interface. We therefore need some guidance in this large parameter space.

For this purpose and in order that this model enlighten us on real systems, we shall consider the case of a $3 M_{\odot}$ star with a one day rotation period. Thus we will use a radius $R=2 \times 10^{9} \mathrm{~m}$, a typical Brunt-Väisälä frequency of $\mathcal{N}=10^{-3} \mathrm{~Hz}$ and a surface gravity of $g=10^{2} \mathrm{~m} / \mathrm{s}^{2}$. The profile of the Brunt-Väisälä frequency needs to reflect more realistic models. In Fig. 1a we plot such a profile for a $3 M_{\odot}$ star at different stages of its evolution on the main sequence. Such profiles should not be taken at face value, especially the contribution of the $\mu$-gradients since only microscopic diffusion is included in this model (produced by the code CESAM, see Morel 1997). In fact it is the aim of the present work to understand the mechanisms by which elements move in the radiative envelope. Therefore we consider the generic situation visualized in Fig. $1 \mathrm{~b}$.

Because of our choice of scalings, the amplitude of nonlinear terms in the momentum equation is independent of rotation and can be appreciated directly from a non-rotating stellar model. In Fig. 2, we display the values of the Rossby number as a function of radius for our typical $3 M_{\odot}$ star. Indeed, the nondimensional function

$\operatorname{Ro}(r)=\frac{\mathcal{N}^{2}(r) r}{4 g(r)}$

gives an interesting approximation of the amplitude of these nonlinear terms. As can be seen, this number is generally less than unity except near the surface layers where it reaches Ro $\sim 5$.

Recalling that $\varepsilon<1$, we see that the other non-linear terms are also less than unity (in fact very much less than unity as shown below). As a first step in the investigation, we ignore them altogether so as to be able to examine the properties of the system with linear equations.

Setting Ro $=\varepsilon=0$, we obtain the system:

$$
\left\{\begin{array}{l}
\boldsymbol{\nabla} \times\left(\boldsymbol{e}_{z} \wedge \boldsymbol{u}-\theta \boldsymbol{r}-E \Delta \boldsymbol{u}\right)=-n_{T}^{2} \sin \theta \cos \theta \boldsymbol{e}_{\varphi} \\
\left(n_{T}^{2} / r\right) u_{r}=\tilde{E}_{T} \Delta \theta \\
\boldsymbol{\nabla} \cdot \boldsymbol{u}=0 .
\end{array}\right.
$$

\section{The asymptotic analysis at $E \ll 1$}

Before solving the full system (6), we first discuss the case of asymptotically small Ekman numbers found in stellar applications.

\subsection{The inviscid profile}

When viscosity is neglected the Eqs. (6) admit a particular solution called (in geophysics) the thermal wind; in our case it reads

$\left\{\begin{array}{l}\boldsymbol{u}^{0}=\left(s \int \frac{n^{2}(r)}{r} \mathrm{~d} r+F(s)\right) \boldsymbol{e}_{\varphi}, \\ \theta=0\end{array}\right.$

where $s=r \sin \theta$ is the radial cylindrical coordinate and $F(s)$ an arbitrary function describing a pure geostrophic solution.

As already pointed out by Busse $(1981,1982)$, the so-called thermal disequilibrium induced by centrifugal acceleration does not imply meridional circulation. A slight differential rotation where the baroclinic torque is balanced by the Coriolis torque gives a steady solution.

However, we also see that such a solution is largely underdetermined since the function $F$ needs to be specified; this degeneracy is lifted by viscosity. The qualitative importance of $F(s)$ comes from the fact that it controls the latitudinal differential rotation. 

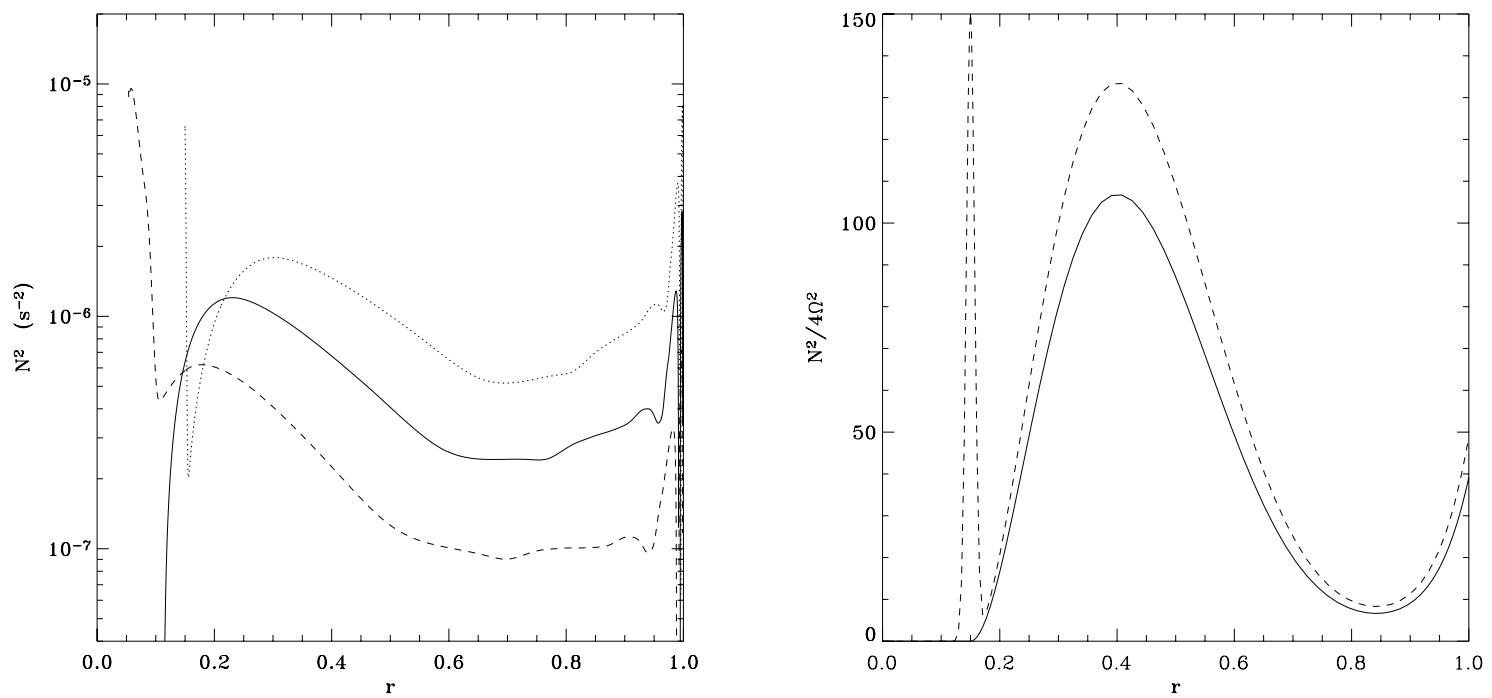

Fig. 1. a) Profiles of the Brunt-Väisälä frequency from the $1 \mathrm{D}$ evolution code CESAM for a $3 M_{\odot}$ star at 0 (solid), $20 \mathrm{Myr}$ (dotted line), $284 \mathrm{Myr}$ (dashed line); these models include microscopic diffusion of Michaud \& Proffitt (1993). b) Adopted profiles for a star with (dashed line) and without (solid line) a $\mu$-barrier.

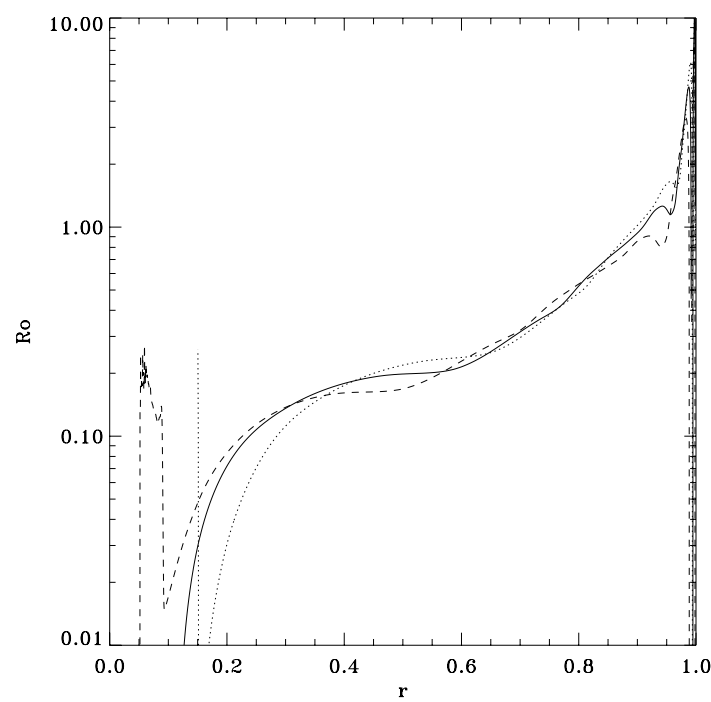

Fig. 2. a) Profiles of the Rossby number $r N^{2}(r) / 4 g(r)$ from the $1 \mathrm{D}$ evolution code CESAM for a $3 M_{\odot}$ star at 0 (solid), 20 Myr (dotted), $284 \mathrm{Myr}$ (dashed).

\subsection{The Ekman boundary layer}

The foregoing thermal wind solution usually does not satisfy the viscous boundary conditions and thus a boundary layer correction needs to be added. Let us review here its main properties.

If $\boldsymbol{u}^{0}$ is the inviscid interior solution and $\tilde{\boldsymbol{u}}$ the boundary layer correction such that $\boldsymbol{u}^{0}+\tilde{\boldsymbol{u}}$ satisfies both the flow equations and the boundary conditions, it is well-known (see Greenspan 1969; Rieutord 1997) that $\tilde{\boldsymbol{u}}$ verifies

$\boldsymbol{e}_{r} \wedge \tilde{\boldsymbol{u}}+\mathrm{i} \tilde{\boldsymbol{u}}=\boldsymbol{C}\left(\boldsymbol{u}^{0}\right) \exp (-\zeta \sqrt{\mathrm{i}|\cos \theta|})$ where $\zeta=(1-r) / \sqrt{E}$ is the radially stretched coordinate and $\boldsymbol{C}$ a complex vector. In the boundary layer the flow is essentially tangential and we may write the full velocity field:

$u_{\theta}+\mathrm{i} u_{\varphi}=C \exp (-\zeta \sqrt{\mathrm{i}|\cos \theta|})+\mathrm{i} u_{\varphi}^{0}$

where the constant $C$ is such that the horizontal stress is zero; namely

$\frac{\partial}{\partial r}\left(\frac{u_{\theta}+\mathrm{i} u_{\varphi}}{r}\right)=0$

which yields

$C \equiv C(\theta)=(1+\mathrm{i}) \sqrt{\frac{E}{2}} \Gamma(\theta)$

with

$\Gamma(\theta)=\frac{F(\sin \theta)-\sin \theta F^{\prime}(\sin \theta)-n^{2}(1) \sin \theta}{\sqrt{|\cos \theta|}}$

where the prime indicates derivatives. From these results we find the meridian velocity in the boundary layer:

$u_{\theta}=\sqrt{\frac{E}{2}} \Gamma(\theta)(\cos \xi+\sin \xi) \mathrm{e}^{-\xi}$

with $\xi=\zeta \sqrt{|\cos \theta| / 2}$.

Such a flow however does not verify mass conservation. This is taken care of by the so-called Ekman pumping which yields the radial component:

$\tilde{u}_{r}=E U(\sin \theta) \cos \xi \mathrm{e}^{-\xi}$

with

$U(s)=n^{2}(1)(1+q(s))+s F^{\prime \prime}(s)+q(s)\left(F^{\prime}-F / s\right)$,

and

$q(s)=1+\frac{s^{2}}{2\left(1-s^{2}\right)}$. 
These expressions show us that near the surface there exists a latitudinal flow $O(\sqrt{E})$ which induces an $O(E)$ circulation in the bulk of the fluid; in turn, this circulation controls the geostrophic flow $F(s) \boldsymbol{e}_{\varphi}$ and thus removes the degeneracy of the thermal wind solution. The boundary layer solution is singular at equator $(\theta=\pi / 2)$; this is the classical equatorial singularity of Ekman layers whose thickness changes to $O\left(E^{2 / 5}\right)$ in a region $O\left(E^{1 / 5}\right)$ around the equator (see also Greenspan 1969).

\subsection{The thermal wind}

Since differential rotation is a major feature of the baroclinic flow, it is useful to push further its analysis and express $F(s)$ as a function of the Brunt-Väisälä frequency profile. For this purpose, we express the meridian circulation as a function of $F$. We thus write the $\varphi$-component of the momentum equation in cylindrical coordinate

$u_{s}=E\left(\Delta-1 / s^{2}\right) u_{\varphi}^{0}$

where $\Delta$ is the Laplacian operator; this equation expresses the local balance between advection and diffusion of angular momentum. It yields

$u_{s}=E\left(F^{\prime \prime}+F^{\prime} / s-F / s^{2}+s C(r)\right)$,

with $\quad C(r)=\left(\frac{n^{2}}{r}\right)^{\prime}+\frac{4 n^{2}}{r^{2}}$.

Mass conservation gives the $z$-component of the flow:

$u_{z}=-\int_{0}^{z} \frac{1}{s} \frac{\partial s u_{s}}{\partial s} \mathrm{~d} z=-E L_{3}(F) z-E \int_{0}^{z} \frac{1}{s} \frac{\partial s^{2} C(r)}{\partial s} \mathrm{~d} z^{\prime}$

with $\quad L_{3}(F)=\frac{1}{s}\left[\left(s F^{\prime}\right)^{\prime \prime}-(F / s)^{\prime}\right]$

while in the integral $r^{2}=z^{\prime 2}+s^{2}$.

Now, $u_{r}=0$ must be satisfied at order $O(E)$ on the outer boundary. Hence, setting $r=1$, we have

$s u_{s}+z u_{z}+\tilde{u}_{r}=0$

which gives the following differential equation for $F$ :

$$
\begin{aligned}
& s F^{\prime \prime}+F^{\prime}-F / s+s^{2} C(1)-\zeta(s)^{2} L_{3}(F) \\
& \quad-\zeta(s) \int_{0}^{\zeta(s)}\left(2 C(r)+s^{2} \frac{C^{\prime}(r)}{r}\right) \mathrm{d} z \\
& \quad+n^{2}(1)(1+q(s))+s F^{\prime \prime}(s)+q(s)\left(F^{\prime}-F / s\right)=0
\end{aligned}
$$

with $\zeta(s)=\sqrt{1-s^{2}}$. This complicated equation is of the form $\mathcal{L}(F)=b$, namely a forced third order differential equation. We do not try to find the general solution of it, but will examine the polynomial solution $F=A s+a s^{3}+b s^{5}$, formally valid at $s \ll 1$, with the hope that since $s \leq 1$, it will give a good idea of the flow.

As expected, the solution is invariant to the addition of a solid rotation; thus $A$ is arbitrary. The first interesting term is $a s^{3}$. Substitution into Eq. (13) gives the following result:

$$
\begin{aligned}
a= & -\frac{1}{2} \int_{0}^{1} \frac{n^{2}(r)}{r^{2}} \mathrm{~d} r \\
b= & \frac{1}{48}\left(\frac{n^{2}}{r}\right)^{\prime}(1)+\frac{19}{192} n^{2}(1) \\
& -\frac{1}{24} \int_{0}^{1}\left\{\frac{n^{2}}{r^{2}}+\frac{1}{3 r}\left[\left(\frac{n^{2}}{r}\right)^{\prime \prime}+4\left(\frac{n^{2}}{r^{2}}\right)^{\prime}\right]\right\} \mathrm{d} r
\end{aligned}
$$

and thus we expect the following differential rotation close to the $z$-axis

$\delta \Omega=\int \frac{n^{2}(r)}{r} \mathrm{~d} r-\frac{s^{2}}{2} \int_{0}^{1} \frac{n^{2}(r)}{r^{2}} \mathrm{~d} r+b s^{4}$

which is now an explicit function of the Brunt-Väisälä frequency profile.

\subsection{The role of stratification}

In the foregoing solution we set $\theta$ to zero and therefore did not bother about stratification. In order to understand its role, we need to evaluate the amplitude of $\theta$, i.e. the scaled temperature fluctuation.

Equation (6b) shows that since $u_{r}$ (i.e. meridian circulation) is $O(E)$, then $\theta$ is $O\left(E / \tilde{E}_{T}\right)$ that is of the order of $\lambda=\mathcal{P} N^{2}$. Thus, in the limit of small $\lambda$, our solution is valid. Stellar fluids are characterized by small Prandtl numbers and usually in rapidly rotating stars one considers that $\lambda \ll 1$ which makes our neglect of buoyancy acceptable.

However, as was pointed out by Spiegel \& Zahn (1970) it is likely that some turbulence is driven by the shear of the baroclinic flow. The viscosity, changed to a turbulent one, is then increased by some factor which therefore also increases the Prandtl number. From the discussion of Zahn (1993), this turbulent Prandtl number may reach values close to unity; hence, even in a rapidly rotating star, the $\lambda$-parameter may be larger than unity (typically up to $10^{2}$ ).

The consequence of such a situation is that a thermal boundary layer appears. Its scale is of course $1 / \sqrt{\lambda}$ and we now assume that $\lambda \gg 1$. Outside this layer, quantities vary on an $O(1)$ scale; noting that $u_{s} \sim u_{r}$, using the $\varphi$-component of the momentum equation together with the heat equation, it follows that

$\theta \sim \lambda u_{\varphi}$

Now, the $\varphi$-component of the vorticity Eq. (6a) shows that in this region

$u_{\varphi} \sim \lambda^{-1}, \quad u_{r} \sim E \lambda^{-1}, \quad$ and $\theta \sim 1$.

Roughly speaking, outside the thermal layer the temperature fluctuation is of order unity and the velocity perturbation is reduced by a factor $O(\lambda)$, while in the thermal layer, radial gradients are increased by a factor $\sqrt{\lambda}$ which gives:

$u_{\varphi} \sim \lambda^{-1}, \quad u_{r} \sim E, \quad$ and $\quad \theta \sim 1$.

Hence, all the dynamics described above for $\mathcal{P} \ll 1$ become squeezed inside the thermal layer with differential rotation reduced by a factor $\lambda$. This is very similar to the solar tachocline where turbulent diffusion inhibits the diffusion of momentum with the help of stratification.

\subsection{Discussion}

The foregoing solution potentially shows very interesting properties of the baroclinic flow; however, it may not be reliable mathematically considering the crudeness of the solution of Eq. (13).

The accuracy of the $a s^{3}$ solution of Eq. (13) may only be estimated by direct comparison to numerical solutions. Anticipating the next section we numerically solve Eq. (6) in the limit of 


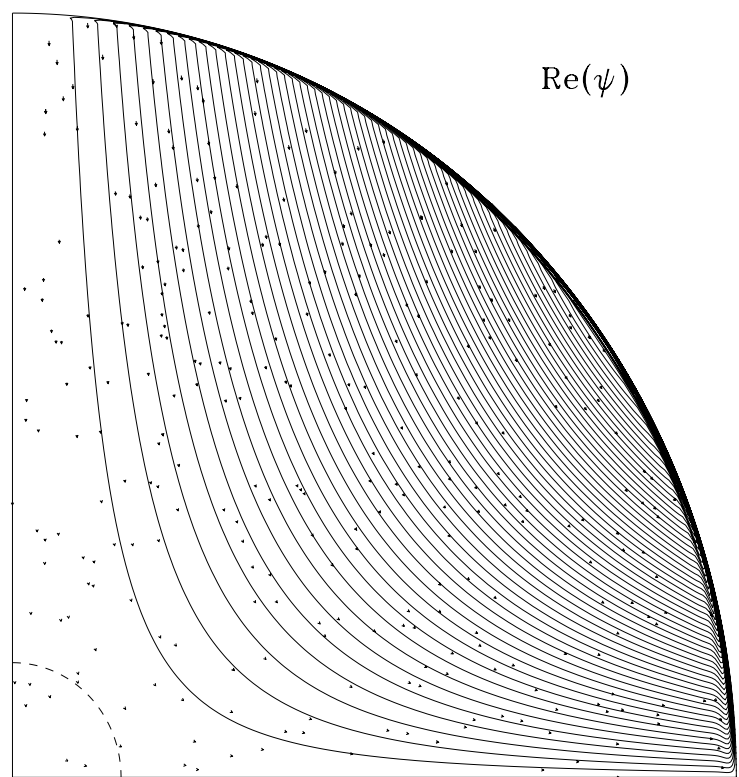

$\mathrm{Nr}=130 \mathrm{~L}=120 \mathrm{E}=1.0 \times 10^{-6} \mathcal{P}=1.0 \times 10^{-4} \mathrm{~N}_{\max }^{2}=1.00 \nu=1.00 \mathrm{CL}=\mathrm{ft}$

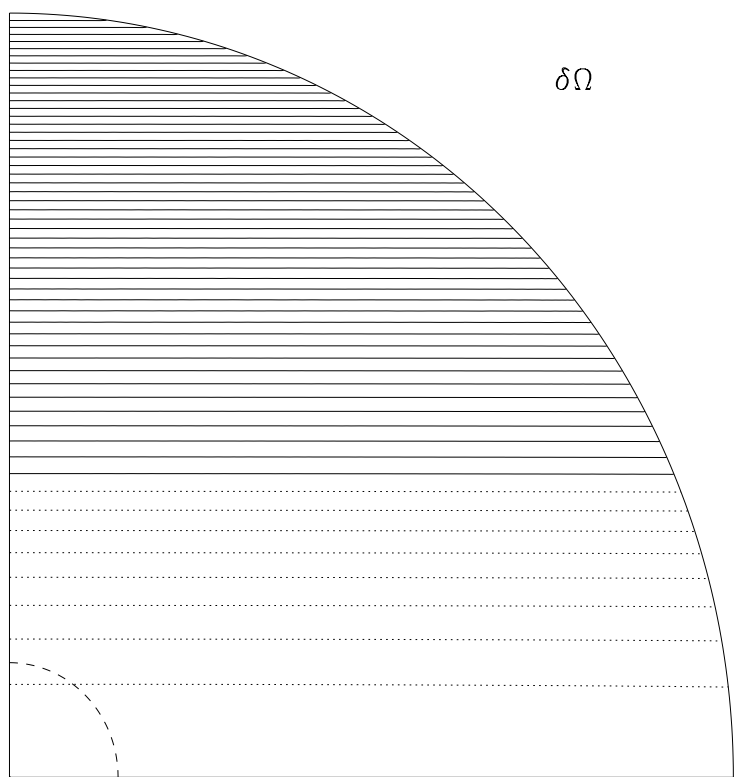

$\mathrm{Nr}=130 \mathrm{~L}=120 \mathrm{E}=1.0 \times 10^{-6} \mathcal{P}=1.0 \times 10^{-4} \mathrm{~N}^{2}{ }_{\max }=1.00 \nu=1.00 \mathrm{CL}=\mathrm{ft}$

Fig. 3. Differential rotation (right) and meridional circulation (left) for a Brunt-Väisälä frequency profile $n(r)=r$.

small Prandtl number. We used a very simple Brunt-Väisälä frequency profile, $n^{2}=r^{2}$, which is standard in the literature (see Chandrasekhar 1961). With such a profile the estimate of the different components of the flow are easy to evaluate $\left(a=-\frac{1}{2}\right.$ and $\left.b=\frac{15}{192}\right)$; as for the differential equation we find

$\delta \Omega=\frac{1}{2} z^{2}$

neglecting $b$; this solution compares extremely well with the numerical solution (see Fig. 3) as soon as $\mathcal{P} \leq 0.1$. It shows that the second term $b s^{5}$ does not much influence the shape of the solution. From the expression of $b$ we see that this is true because, in this case, $n^{2}$ is a smooth function of $r$. In stellar situations, the coefficient $\left(n^{2} / r\right)^{\prime}(1)$ might be very large if sharp gradients develop in the upper layers of the star.

Reassured by these computations, Eq. (14) therefore shows a very interesting result: if the Brunt-Väisälä frequency profile of a star is sufficiently smooth, the latitudinal differential rotation in a stably stratified envelope driven by the sole baroclinicity is a fast rotating pole and a slower rotating equator. Moreover, the $a$-coefficient being an integral over the star, it is not sensitive to the detailed shape of the Brunt-Väisälä frequency profile and therefore is a rather robust quantity.

Conversely, in a convecting envelope, with viscous-like Reynolds stresses, baroclinic torques induce a fast rotating equator and slow poles, like the sun actually. Of course, in the solar case, this may just be a coincidence since baroclinicity in the convective zone of the sun does not come from a centrifugal effect but rather from the convection itself (see Brun \& Toomre 2002). Nevertheless, in rapidly rotating (i.e. young) solar type stars, our result shows that baroclinicity drives a solar-like differential rotation.

Finally, the solution also gives the form of the meridian circulation through Eqs. (11) and (12). For instance the number of cells can be retrieved from the expression of $u_{s}$. At equator, $s=r$ and $u_{s}$ reads

$u_{s}=E\left(\frac{1}{r^{3}} \frac{\mathrm{d}\left(r^{3} n^{2}\right)}{\mathrm{d} r}-4 r \int_{0}^{1} \frac{n^{2}}{r^{2}} \mathrm{~d} r\right)$.
The number of zeros of this function, plus one, gives the number of cells according to the Brunt-Väisälä frequency profile. The expression of $u_{\theta}$ in the Ekman layer (9) shows that at the surface, $u_{\theta} \sim-n^{2}(1) s$, which means that the flow is poleward. If the Brunt-Väisälä frequency profile is smooth enough this motion extends to low latitudes. However, because of the $\sin \xi+\cos \xi$ dependence, the boundary layer flow reverses just below the surface at $r=1-\frac{3 \pi}{4} \sqrt{\frac{2 E}{|\cos \theta|}}$.

From the amplitude of the meridian flow, one can estimate the circulation time scale which is:

$T_{\text {circ }}=\frac{2 \Omega}{\mathcal{N} F r} T_{\text {visc }}=\frac{T_{\text {visc }}}{\text { Ro }}$.

Since Ro $\sim 1$, this time scale is of the order of the viscous diffusion time scale $T_{\text {visc }}$ which is very large if only microscopic viscosity is diffusing momentum.

\section{The general case}

We now turn to a more complex model with which we analyse the effects of a convective core and of $\mu$-gradients. The inclusion of $\mu$-gradients amounts to the addition of the equation of a concentration $c$ and the modification of the buoyancy term; the linear system (6) needs to be changed into:

$$
\left\{\begin{array}{l}
\boldsymbol{\nabla} \times\left(\boldsymbol{e}_{z} \wedge \boldsymbol{u}-(\theta-c) \boldsymbol{r}-E \Delta \boldsymbol{u}\right)=-n^{2} \sin \theta \cos \theta \boldsymbol{e}_{\varphi} \\
\left(n_{T}^{2} / r\right) u_{r}=\tilde{E}_{T} \Delta \theta \\
-\left(n_{\mu}^{2} / r\right) u_{r}=\tilde{E}_{\mathrm{c}} \Delta c \\
\boldsymbol{\nabla} \cdot \boldsymbol{u}=0
\end{array}\right.
$$

where we introduced $n^{2}=n_{T}^{2}+n_{\mu}^{2}$ and $\tilde{E}_{\mathrm{c}}=\left(D_{\mathrm{c}} / 2 \Omega R^{2}\right) \times$ $(2 \Omega / \mathcal{N})^{2}$ with $D_{\text {c }}$ being the diffusivity of element " $c$ ". This element is supposed to be heavier than the surrounding gas.

Since solutions are much more complicated we use numerical solutions and first describe the numerical technique. 


\subsection{The numerical method}

To solve the system (15) we first project the variables onto the spherical harmonic base (see Rieutord 1987)

$\boldsymbol{u}=\sum_{\ell=0}^{+\infty} \sum_{m=-\ell}^{+\ell} u_{m}^{\ell} \boldsymbol{R}_{\ell}^{m}+v_{m}^{\ell} \boldsymbol{S}_{\ell}^{m}+w_{m}^{\ell} \boldsymbol{T}_{\ell}^{m}$,

$\theta=\sum_{\ell=0}^{+\infty} \sum_{m=-\ell}^{+\ell} \theta_{m}^{\ell} Y_{\ell}^{m}, \quad c=\sum_{\ell=0}^{+\infty} \sum_{m=-\ell}^{+\ell} c_{m}^{\ell} Y_{\ell}^{m}$

where

$\boldsymbol{R}_{\ell}^{m}=Y_{\ell}^{m} \boldsymbol{e}_{r}, \quad \boldsymbol{S}_{\ell}^{m}=\boldsymbol{\nabla} Y_{\ell}^{m}, \quad \boldsymbol{T}_{\ell}^{m}=\boldsymbol{\nabla} \times \boldsymbol{R}_{\ell}^{m}$

with $Y_{\ell}^{m}$ being the usual normalized spherical harmonic function. Since the flow is divergenceless, $v_{m}^{l}$ may be expressed as:

$v_{m}^{\ell}=\frac{1}{\ell(\ell+1)} \frac{1}{r} \frac{\mathrm{d} r^{2} u_{m}^{\ell}}{\mathrm{d} r}$

Then, projecting the equations (the vorticity equation needs be projected only on $\boldsymbol{R}_{\ell}^{m}$ and $\boldsymbol{T}_{\ell}^{m}$ ), we get

$$
\left\{\begin{array}{c}
E \Delta_{\ell} w_{m}^{\ell}+A(\ell) r^{\ell-1} \frac{\partial}{\partial r}\left(\frac{u_{m}^{\ell-1}}{r^{\ell-2}}\right)+A(\ell+1) r^{-\ell-2} \frac{\partial}{\partial r}\left(r^{\ell+3} u_{m}^{\ell+1}\right)=0 \\
E \Delta_{\ell} \Delta_{\ell}\left(r u_{m}^{\ell}\right)=B(\ell) r^{\ell-1} \frac{\partial}{\partial r}\left(\frac{w_{m}^{\ell-1}}{r^{r-1}}\right)+B(\ell+1) r^{-\ell-2} \frac{\partial}{\partial r}\left(r^{\ell+2} w_{m}^{\ell+1}\right) \\
\quad+\ell(\ell+1)\left(\theta_{m}^{\ell}-c_{m}^{\ell}\right)-\left(n_{T}^{2}+n_{\mu}^{2}\right) N_{2} \delta_{\ell 2} \\
\tilde{E}_{T} \Delta_{\ell} \theta_{m}^{\ell}-n_{T}^{2} / r u_{m}^{\ell}=0 \\
\tilde{E}_{\mathrm{c}} \Delta_{\ell} c_{m}^{\ell}+n_{\mu}^{2} / r u_{m}^{\ell}=0
\end{array}\right.
$$

where $N_{2}=\sqrt{\frac{16 \pi}{5}}$ and

$$
A(\ell)=\frac{1}{\ell^{2}} \sqrt{\frac{\ell^{2}-m^{2}}{(2 \ell-1)(2 \ell+1)}}, \quad B(\ell)=\left(\ell^{2}-1\right) \ell^{2} A(\ell) .
$$

This coupled system of differential equation needs to be completed by boundary conditions at the surface of the sphere (at the centre we only demand the regularity of the solutions). Concerning the velocity, we impose stress-free conditions, thus

$u_{m}^{\ell}=0, \quad \frac{\mathrm{d}^{2} r u_{m}^{\ell}}{\mathrm{d} r^{2}}(1)=0, \quad \frac{\mathrm{d}}{\mathrm{d} r}\left(\frac{w_{m}^{\ell}}{r}\right)=0$

while on the temperature and concentration we impose respectively no fluctuation and no flux at $r=1$, namely

$\theta_{m}^{\ell}=0, \quad \frac{\mathrm{d} c_{m}^{\ell}}{\mathrm{d} r}=0$.

Further, we need interface conditions at the core-envelope boundary. These conditions express the continuity of the velocity, temperature, concentration, fluxes and stress fields. The continuity of first quantities simply translates as the continuity of

$u_{m}^{\ell}, \quad \frac{\mathrm{d} u_{m}^{\ell}}{\mathrm{d} r}, \quad w_{m}^{\ell}, \quad \theta_{m}^{\ell}, \quad c_{m}^{\ell}$ while the continuity of fluxes and stresses must reflect the changes in the transport coefficients between the turbulent convective core and the radiative envelope. Continuity of the three components of stress demands the continuity of

$$
\begin{aligned}
& E\left(r \frac{\mathrm{d}^{2} u_{m}^{\ell}}{\mathrm{d} r^{2}}+2 \frac{\mathrm{d} u_{m}^{\ell}}{\mathrm{d} r}+(\ell(\ell+1)-2) u_{m}^{\ell} / r\right) \\
& E\left(\frac{\mathrm{d} w_{m}^{\ell}}{\mathrm{d} r}-\frac{w_{m}^{\ell}}{r}\right)
\end{aligned}
$$

$p_{m}^{\ell}-2 E \frac{\mathrm{d} u_{m}^{\ell}}{\mathrm{d} r} \quad$ or $\quad E\left(\frac{\mathrm{d}^{3} u_{m}^{\ell}}{\mathrm{d} r^{3}}+\frac{6}{r} \frac{\mathrm{d}^{2} u_{m}^{\ell}}{\mathrm{d} r^{2}}+\frac{3(2-\ell(\ell+1))}{r^{2}} \frac{\mathrm{d} u_{m}^{\ell}}{\mathrm{d} r}\right)$

while continuity of heat flux and concentration flux impose the continuity of

$\tilde{E}_{T} \frac{\mathrm{d} \theta_{m}^{\ell}}{\mathrm{d} r} \quad$ and $\quad \tilde{E}_{\mathrm{c}} \frac{\mathrm{d} c_{m}^{\ell}}{\mathrm{d} r}$

\subsection{The shape of the baroclinic flow}

We investigate the baroclinic flow which is represented by both the differential rotation and the associated meridian circulation. We focus our attention on the influence of the main uncertainties of the problem, namely the sensitivity to viscous transport and the height of the $\mu$-barrier.

\subsubsection{With no jump in viscosity}

We first consider a simple configuration where we set a BruntVäisälä frequency profile with no $\mu$-barrier, no viscosity jump at core-envelope interface. We thus find a plain baroclinic flow and can check the agreement with our foregoing theoretical results. The meridian circulation is shown in Fig. 4 together with the associated differential rotation. The plot of the angular velocity shows the typical shape of the thermal wind solution given by Eq. (7). This shape remains identical if the viscosity is decreased (i.e. with lower Ekman or Prandlt numbers). This flow is essentially azimuthal with faster rotating poles as predicted above. The meridian circulation is much weaker (see Fig. 5), being essentially $O(E)$. As expected from the above boundary layer analysis, near the surface the latitudinal component increases very strongly by a factor $E^{-1 / 2}$ (this is an effect of mass conservation). A detailed view of the velocity profiles in the Ekman layer is given in Fig. 6.

To have further information on the baroclinic flow we examined the case where the Prandtl number is of order unity. In that case the value of $\lambda$ is important and we set it to 100 according to our typical rotating star. The meridian circulation along with the radial profiles of the velocity field are shown in Fig. 7. Obviously, the circulation has much decreased. This is a direct consequence of the heat Eq. (6b) which imposes a reduction of radial velocity when heat diffusion decreases; as a consequence, advection of momentum is less and therefore diffusion of momentum must decrease, which is obtained by reducing the differential rotation as shown in Fig. $7 b$.

\subsubsection{With a viscosity jump at the core-envelope boundary}

To look for more realistic models, we now consider the effect of the viscosity jump at the core-envelope boundary which is 


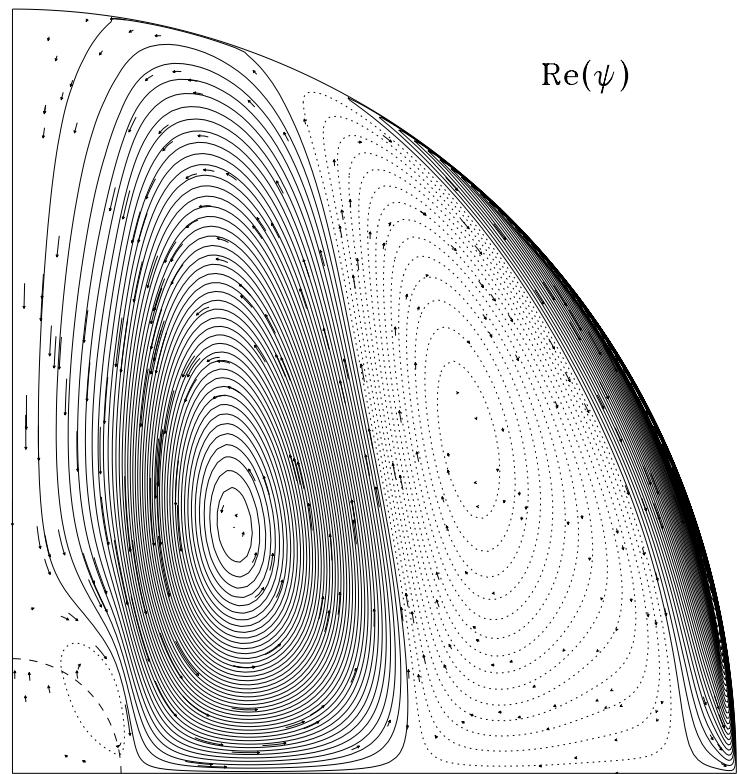

$\mathrm{Nr}=130 \mathrm{~L}=120 \mathrm{E}=1.0 \times 10^{-6} \mathscr{P}=1.0 \times 10^{-4} \mathrm{~N}_{\max }^{2}=1.00 \nu=1.00 \mathrm{CL}=\mathrm{ft}$

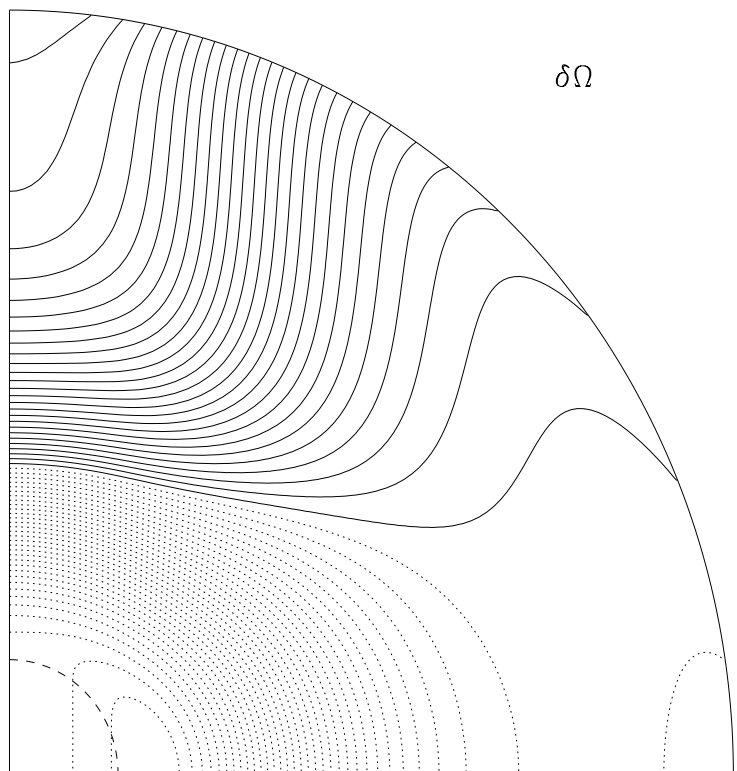

$\mathrm{Nr}=130 \mathrm{~L}=120 \mathrm{E}=1.0 \times 10^{-6} \mathcal{P}=1.0 \times 10^{-4} \mathrm{~N}_{\max }^{2}=1.00 \nu=1.00 \mathrm{CL}=\mathrm{ft}$

Fig. 4. Left: the meridional circulation of the baroclinic flow generated by the Brunt-Väisälä frequency profile in Fig. $1 \mathrm{~b}$ (solid curve) for a fluid with constant viscosity. Right: the associated differential rotation showing the fast rotating pole and slow equator (solid lines are for positive contours, dotted lines for negative ones).

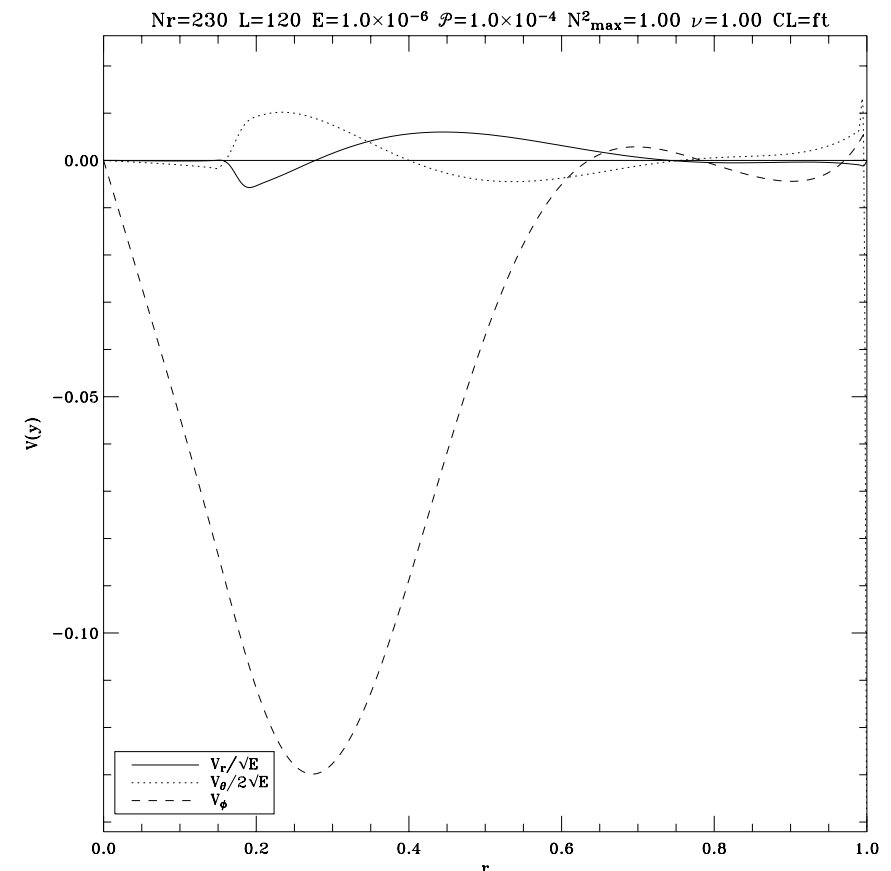

Fig. 5. Radial profile at $\theta=1$ rd of the velocity components of the baroclinic flow. Note that the $v_{r}$ and $v_{\theta}$ have been multiplied by a factor $O\left(E^{-1 / 2}\right) \gg 1$.

brought about by turbulent convection in the core. The convective core is thus considered as a much more viscous fluid with negligible stratification (Brunt-Väisälä frequency is set to zero).

The interesting result is that the meridian circulation is strongly modified, as shown in Fig. 8. This figure shows that the jump in the mean mechanical properties of the fluids generates a shear layer parallel to the axis of rotation. Such a layer is a Stewartson layer, well-known in the dynamics of rotating fluids. The dynamics of these layers is controlled by a delicate balance between viscous stress, pressure gradient and the Coriolis

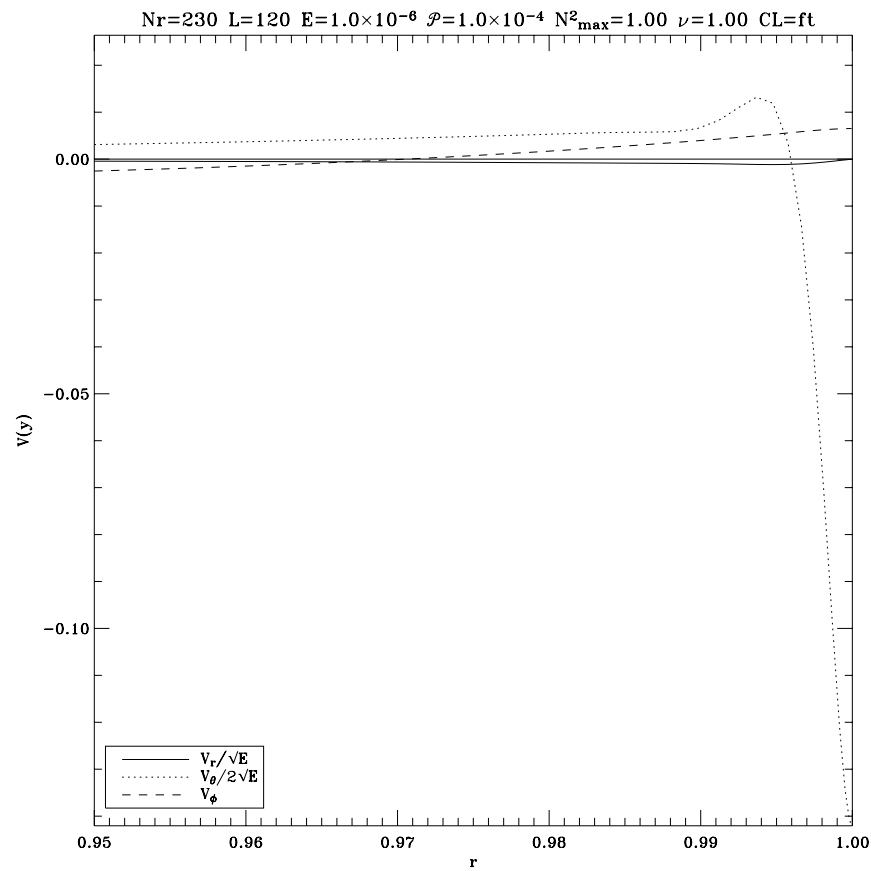

Fig. 6. Same as Fig. 5, but with emphasis on the Ekman layer.

acceleration. As shown by Stewartson (1966), such layers are nested layers whose width scales with $v^{1 / 3}, v^{1 / 4}$ or $v^{2 / 7}$. However, while the properties of such layers are well known in the simple case of incompressible fluids, they remain unexplored when the fluid is stably stratified like here.

The interesting point of this feature is that the scale of the meridian flow is much reduced in one direction; the scale controlling the shear layer pumping is $O\left(E^{1 / 4}\right)$ if we follow Stewartson theory. This means that the circulation time is 


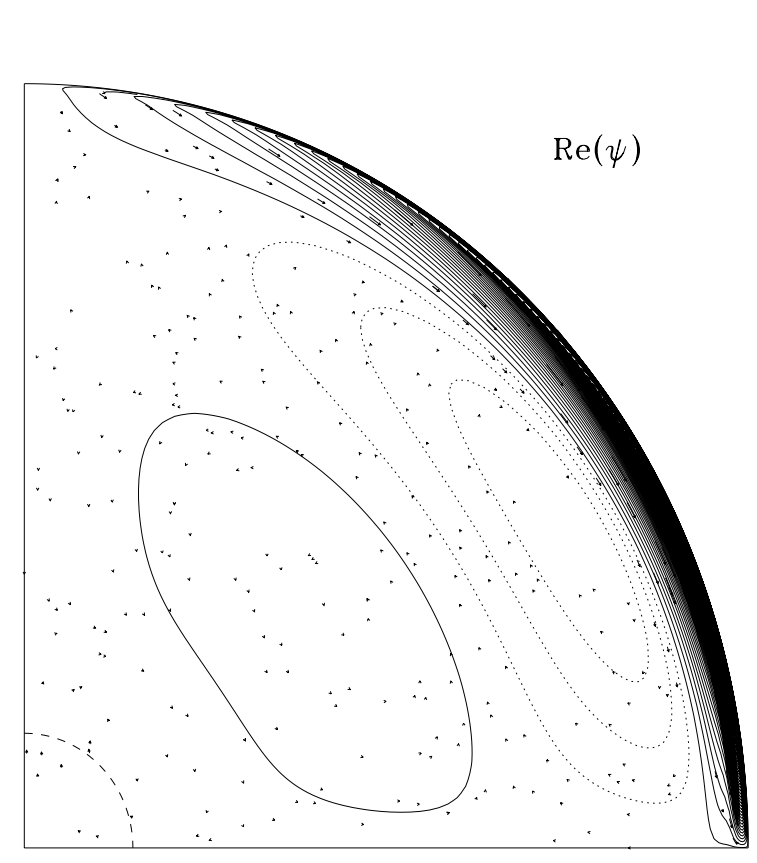

$\mathrm{Nr}=130 \mathrm{~L}=120 \mathrm{E}=4.0 \times 10^{-6} \stackrel{\mathcal{P}}{=}=1.00 \mathrm{~N}_{\text {max }}^{2}=1.0 \times 10^{2} \quad \nu=1.00 \quad \mathrm{CL}=\mathrm{ft}$

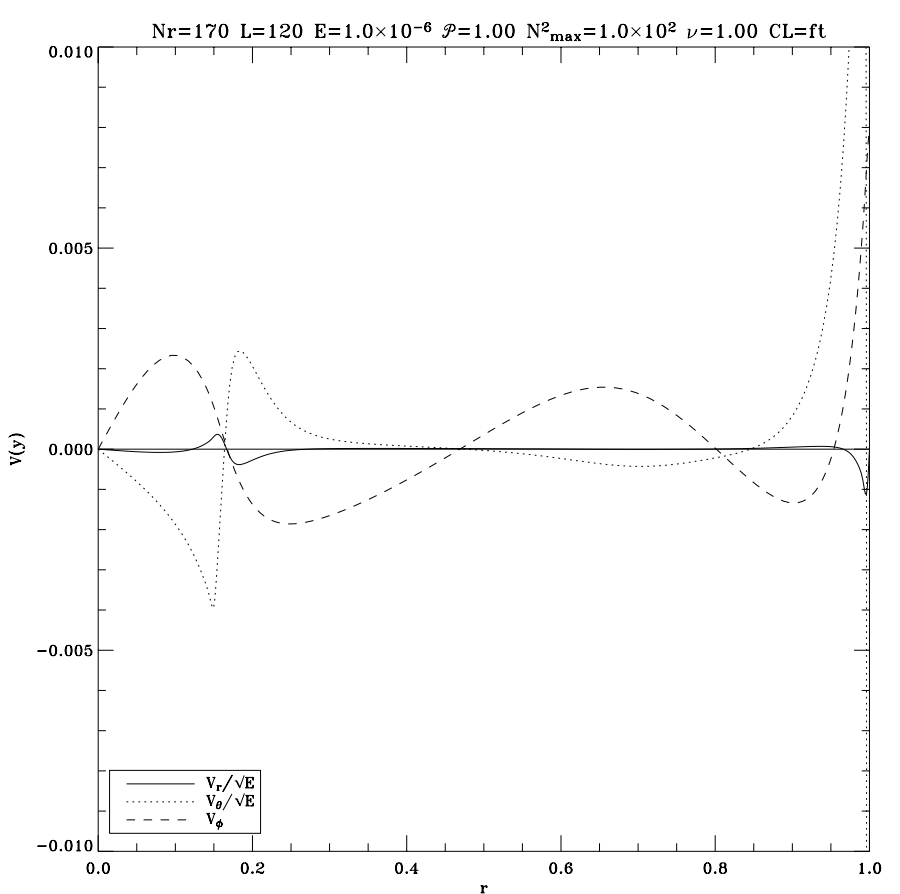

Fig. 7. Left: same as Fig. 4a but with $\mathcal{P}=1$. Right: the associated velocity profiles. Note the squeezed circulation in the thermal layer which width is $\sim 0.1$, and the reduction of the azimuthal velocity in the bulk of the domain.

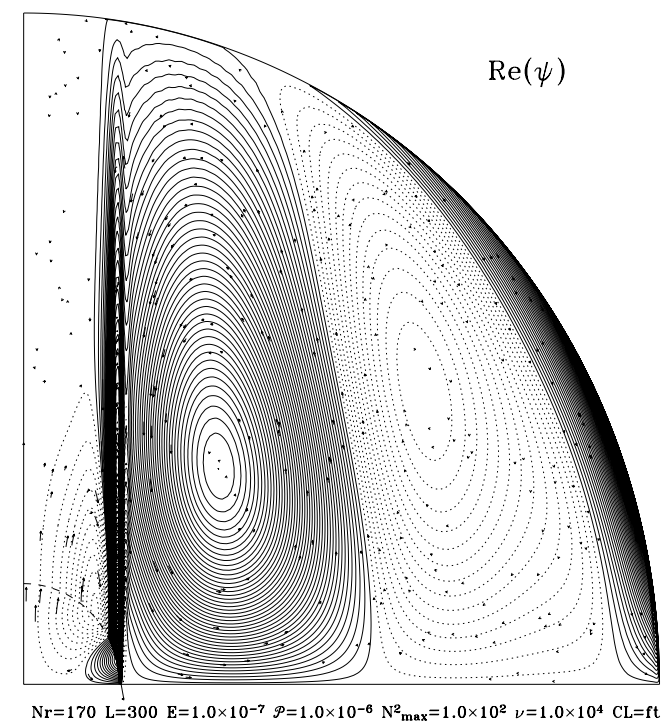

Fig. 8. Same as Fig. 4 but the core viscosity is $10^{4}$ higher than that of the envelope.

reduced by a factor $O\left(E^{1 / 4}\right)$. Indeed, mass conservation implies that

$\frac{\partial V_{\perp}}{\partial x_{\perp}}=-\frac{\partial V_{/ /}}{\partial x_{/ /}}$

where $V_{\perp}$ is the pumping induced by the shear layer and which should be identified as the meridian flow (see Fig. 9). Since $\partial / \partial x_{\perp} \sim E^{-1 / 4}$ and $\partial / \partial x_{/ /} \sim 1, V_{/ /} \sim E^{-1 / 4} V_{\perp}$; hence the advection time from core to surface is reduced by a factor $O\left(E^{1 / 4}\right)$ which is quite small as shown below.

Will this dynamical feature resist to the building of $\mu$-barriers during the evolution of the star? To answer this question, we computed a model with a $\mu$ gradient as shown in Fig. $1 \mathrm{~b}$ (dashed line); namely the height of the $\mu$-barrier has been raised such

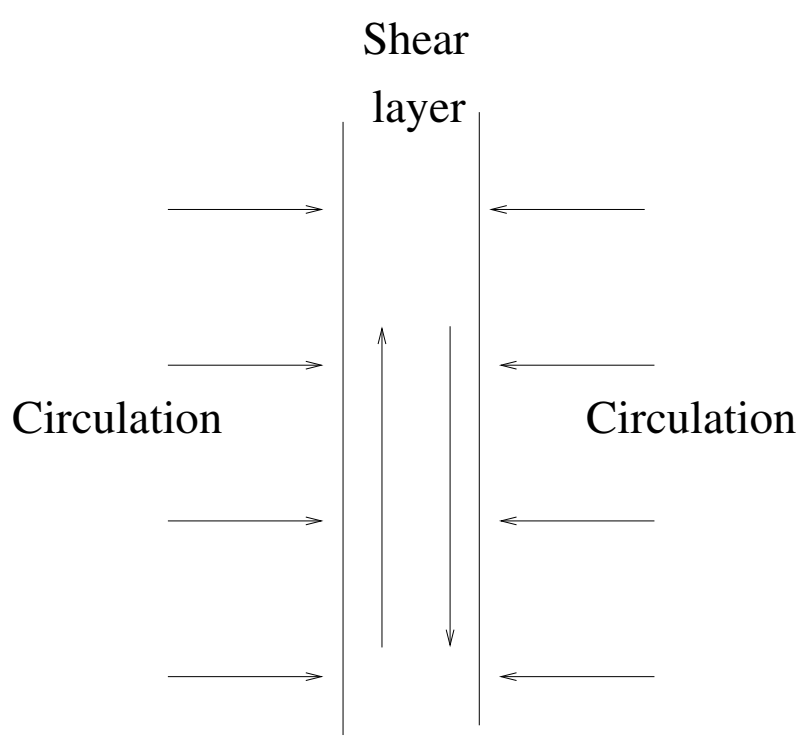

Fig. 9. Schematic view of the Stewartson layer.

that $N_{\mu}^{2} \sim N_{T}^{2}$. We assumed that elemental diffusivity is of the same order of magnitude as the viscosity, i.e. the Schmidt number $v / D_{\mathrm{c}}$ is of order unity. Figure 10 shows the result: the Stewartson layer has disappeared. It is replaced by a meridional flow within the core.

\subsection{Stability}

After the computation of the global baroclinic flow, we may wonder whether it is stable or not. This question is not an easy one since many instabilities are possible (see Knobloch \& Spruit 1982), but we can discuss it qualitatively.

A first class of instabilities are the barotropic ones; they do not take advantage of the baroclinic state and are basically due to 


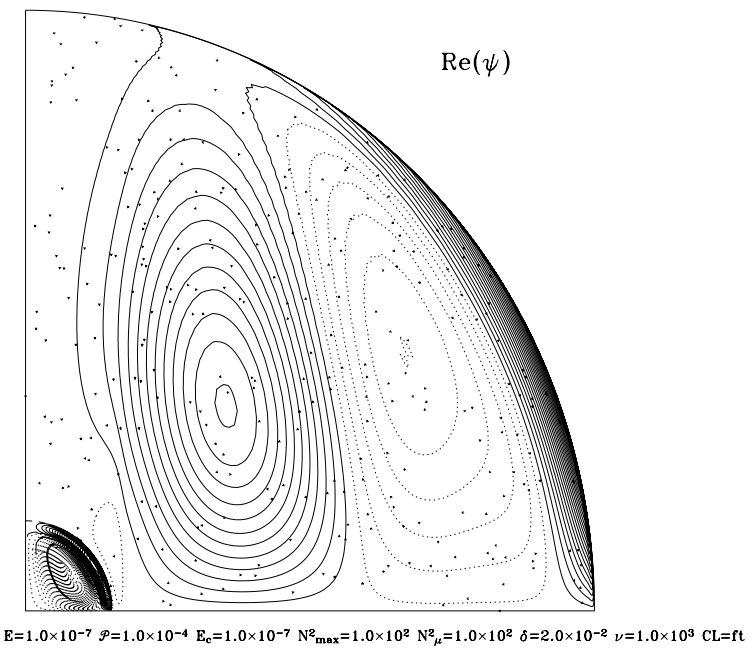

Fig. 10. Same as Fig. 8 but with a $\mu$-barrier where $N_{\mu}^{2}=N_{T}^{2}=100$. Note the disappearance of the Stewartson layer.

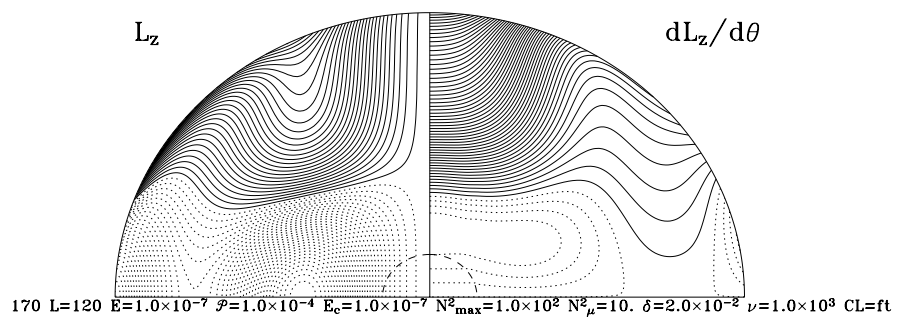

Fig. 11. Angular momentum distribution and its derivative. The dotted lines in $\partial L_{z} / \partial \theta$ show regions where the barotropic axisymmetric instability may develop (solid lines are for positive contours, dotted lines for negative ones).

the shear. The axisymmetric instability is also known as the centrifugal instability and is controlled by the angular momentum profile. The Rayleigh criterion, taking into account the stable stratification, defines the onset of instability as

$\frac{\partial L_{z}}{\partial s}<0 \quad$ at $r=$ const.,$\quad \Longleftrightarrow \quad \frac{1}{\cos \theta} \frac{\partial L_{z}}{\partial \theta}<0$

that is, when the angular momentum decreases with the cylindrical radius. The constraint $r=$ const. avoids the stabilizing effect of the Brunt-Väisälä frequency profile.

For our system the angular momentum of the flow is

$L_{z}=\Omega s^{2}+s V_{\varphi}=\Omega R^{2}\left(s^{2}+2 \operatorname{Ro} s \bar{u}_{\varphi}\right)$

so that the flow is unstable if

$r \cos \theta+\operatorname{Ro} \sum_{\ell} \ell(\ell+1) w_{m}^{\ell} Y_{\ell}^{m}<0$

This formula shows that the Rossby number controls this instability. As discussed above, this parameter is in the range [0.1, $10]$ in our reference star. We find that for Ro $\lesssim 3$ the flow is stable and, as shown in Fig. 11, equatorial regions at destabilized first when Ro increases. We shall not push further the analysis since at this point only a detailed analysis would give sensible results. We just conclude that in view of Fig. 2, we expect such instability only in equatorial surface layers.
The other barotropic instability is the non-axisymmetric shear instability. Basically, in a stably stratified fluid it is controlled by the Richardson number, e.g.

$\mathrm{Ri}=\frac{\mathcal{N}^{2}}{\left(\mathrm{~d} v_{\varphi} / \mathrm{d} r\right)^{2}}=\mathrm{Fr}^{-2}$.

In our case this number appears to scale with the inverse square of the Froude number which we found of order unity. The largescale flow is most likely stable and a rigorous stability analysis is needed to give precise answers in stellar conditions. Nevertheless, following Zahn (1993), this flow may still destabilize scales small enough that they are insensitive to the stable stratification because of the large heat diffusion coefficient. The instability of small scales might thus provide the rotating radiative zone with some turbulence and hence some turbulent viscosity. Zahn (1993) shows that such turbulent viscosity is within the range

$\operatorname{Re}_{\mathrm{c}} v \leq v_{T}=\ell^{2} \frac{\mathrm{d} v_{\varphi}}{\mathrm{d} r} \leq \frac{\kappa \mathrm{Ri}_{\mathrm{c}}}{\mathcal{N}^{2}}\left(\frac{\mathrm{d} v_{\varphi}}{\mathrm{d} r}\right)^{2}$

where $\mathrm{Re}_{\mathrm{c}}$ is the critical Reynolds number for shear flow, typically around 1000 , and $\mathrm{Ri}_{\mathrm{c}}$ the critical Richardson number for stably stratified shear flow, around 0.25 . These bounds on the turbulent viscosity show that the viscosity is increased at least by a factor $\operatorname{Re}_{\mathrm{c}}$ and at maximum by a factor $O\left(\mathcal{P}^{-1}\right)$. This raises the Ekman number from $10^{-18}$ to the range $\left[10^{-15}, 10^{-12}\right]$ which is still very small. Moreover, the Prandtl number is increased by a factor between $\operatorname{Re}_{\mathrm{c}}$ and $\mathcal{P}^{-1}$ which means, in the latter case, an effective Prandtl number of order unity and a $\lambda$-number significantly larger than unity. The turbulence is likely anisotropic and the values of the effective Prandtl number depend whether one considers horizontal or vertical diffusion. An effective Prandtl number of unity is certainly an upper bound.

Besides these classical instabilities (for fluid flows), there are also baroclinic instabilities which are specific to this type of flow driven by the baroclinicity. As discussed by Spruit \& Knobloch (1984), one should separate the true baroclinic instability which develops because a fluid parcel may move in a direction between entropy levels and isobars, and diffusive instabilities (GoldreichSchubert-Fricke and ABCD) which take advantage of double diffusion. These instabilities, specific to baroclinic flows, are rather weak and one question is whether they would persist if the diffusivities are increased by some turbulence generated by the barotropic ones. More work is needed to characterize them, especially in a global approach.

\section{Discussion}

\subsection{Stellar numbers}

Our test star is a $3 M_{\odot}$ object rotating with a period of one day. On the ZAMS, its radius is $2.6 R_{\odot}=2 \times 10^{9} \mathrm{~m}$. The typical kinematic viscosity of the plasma is $v=10^{-3} \mathrm{~m}^{2} / \mathrm{s}$ yielding an Ekman number of $E \sim 2 \times 10^{-18}$ which is very small. The corresponding viscous time is $T_{\text {visc }}=R^{2} / v \sim 10^{14} \mathrm{yr}$. The circulation time is typically a viscous time:

$T_{\text {circ }}=\frac{R}{v_{r}} \sim \frac{1}{\operatorname{Fr} \mathcal{N} \boldsymbol{u}_{\varphi}} \sim \frac{T_{\text {visc }}}{\operatorname{Ro}}$

since Ro $\sim 1$. As we discussed above, various instabilities may trigger turbulence and hence raise the viscosity to turbulent values. From the typical value of the microscopic Prandtl number, 
namely $10^{-6}$, and that of critical Reynolds numbers, $\sim 10^{3}$, we can expect an increase of the Ekman number to $2 \times 10^{-15} \rightarrow$ $2 \times 10^{-12}$ and hence a reduction of the circulation time to $10^{11} \rightarrow 10^{8} \mathrm{yr}\left(10^{8} \mathrm{yr}\right.$ is the Kelvin-Helmoltz time of our model). In the early phase of evolution, when $\mu$-barriers are not strong enough, this time scale may be further reduced by the presence of the Stewartson layer. However, this layer is sensitive to the $\lambda$-number, which means sensitive to the Prandtl number. Thus, if turbulence is strong enough to raise the Prandtl number to order unity values, the Stewartson layer disappears. If it is mild enough and increases the viscosity by a factor $10^{3}$ only, the Stewartson layer has a thickness $O\left(2 \times 10^{-4}\right)$ which reduces the circulation time further by a factor of $5 \times 10^{3}$; thus, mild turbulence and Stewarson layer may reduce the circulation time by a factor of $5 \times 10^{6}$ leaving a time scale for partial mixing of around $10^{7} \mathrm{yr}$. On the other hand if turbulence is strong, circulation outside the thermal layer, which reaches the core, is $\sim \mathcal{P}^{-1}$ faster thanks to the increased viscosity, but $\lambda^{-1}$ slower because of stratification. However, the $\lambda^{-1}$ factor only reduces the advection time; if turbulence is strong, elements may diffuse just like momentum and the time scale is $T_{\text {visc }} \mathcal{P}=10^{8} \mathrm{yr}$.

Rather than the numbers, the role played by the different hydrodynamical processes in controlling the transport of element is important. These processes may control each other: a mild turbulence being helped by a strong Stewartson layer yields a mixing time scale not much different from the one derived in the strong turbulence case. This leaves the possibility that mixing may not be too sensitive to the hydrodynamical details. In particular, we do not include angular momentum loss through a stellar wind (see Zahn 1992); our result shows the possible mixing occuring in the weak wind limit.

We now examine the differential rotation induced by baroclinicity. The scalings give the following relation:

$\frac{\Delta \Omega}{\Omega}=2 \operatorname{Ro} \delta \Omega$

where $\delta \Omega=u_{\varphi} / s$. The numerical solutions show that $\delta \Omega_{\text {pole }}-$ $\delta \Omega_{\mathrm{eq}} \sim 0.5$ when $\mathcal{P} \ll 1$ and decreases to $0.01=O\left(\lambda^{-1}\right)$ when $\mathcal{P} \sim 1$.

From these values we see that such a model predicts a large differential rotation when the Prandtl number is small and a strong reduction of it if some turbulence develops (and behaves as a Newtonian fluid!).

\subsection{Non-linear terms}

What is the importance of the non-linear terms that we neglected? They are Ro $(\boldsymbol{u} \cdot \nabla \boldsymbol{u})$ and $\varepsilon \boldsymbol{u} \cdot \boldsymbol{\nabla} \theta$. Neglecting $O(E)$ terms, the momentum nonlinear term is just $-\operatorname{Ro} u_{\varphi}^{2} / s \boldsymbol{e}_{\varphi}$, i.e. a centrifugal correction to that of the background rotation. We thus expect a slight modification of the baroclinicity but no qualitatively important change.

Concerning the heat advection, the weakness of meridional circulation $O(E)$, plus the $O(\lambda)$ amplitude of $\theta$, make this nonlinear term $O(\varepsilon \lambda E)$, thus extremely small and negligible.

Thus nonlinear terms are either very small or do not bring new physical phenomena in the steady solution. Taking into account the initial assumption of the Boussinesq approximation, refining the solution with these terms is unnecessary.

\subsection{The Boussinesq approximation}

In a more realistic approach, taking into account density variations of the background, would our results persist? The
Boussinesq approximation is indeed the consequence of two limits: the fluid velocity is small compared to the sound velocity and the density scale height is large compared to the size of the container (see Rieutord 1997). The first constraint is easily met: Mach numbers are small. The second one is not because the density varies on a scale comparable to the size of the star. Thus, the simplification of the equation of mass conservation from $\boldsymbol{\nabla} \cdot \rho \boldsymbol{v}=0$ into $\boldsymbol{\nabla} \cdot \boldsymbol{v}=0$ is inappropriate and quantitatively our model should be taken with care. Qualitatively, however, density variations cannot modify the hydrodynamical features like the sign of the differential rotation, the appearance of Stewartson layer, etc. The use of the momentum $\rho \boldsymbol{v}$ instead of $v$ would solve this problem, but then would be inconsistent with the neglect of the effects of centrifugal distortion. Thus, even if the use of the Boussinesq approximation seems exagerated for a star, it provides an interesting view of its hydrodynamics: it takes into account all the force fields that control the flows and gives a physically self-consistent model.

\subsection{Conclusions}

To end this paper we would like to stress the most interesting points of this model, namely:

- The determination of the differential rotation in radiative envelope as a function of the Brunt-Väisälä frequency profile and its sensitivity to the amplitude of the turbulent viscosity.

- The demonstration that, when the Brunt-Väisälä frequency varies on a scale of the order of the radius, baroclinicity generates a fast rotating pole and slow equator and the opposite in the case the envelope is convective.

- The determination of the meridian circulation, its shape and amplitude, its sensitivity to Prandtl number and BruntVäisälä frequency profile.

- The appearance of Stewartson layers induced by the jump in the mechanical properties of the fluid at the core-envelope boundary and the sensitivity of this layer to the $\mu$-gradients or the Prandtl number.

- The richness of the dynamics of these envelopes and the compensation, as far as mixing is concerned, which may result from large-scale flows (Stewartson layer) and smallscale turbulence.

Naturally, such a model cannot pretend to describe actual stars at face value. It should rather be viewed as a laboratory experiment aimed at studying some aspects of the stellar dynamics. Its (relative) simplicity indeed authorizes the study of hydrodynamical instabilities at global scale through either linear analysis or full numerical simulations. For instance, one may think to use it to study dynamo effect in radiative envelope following Braithwaite (2006) and to study the effects of an additional driving like the angular momentum loss generated by a wind.

The next step is to compute the same flow in the real, spheroidal geometry of rapidly rotating stars. Such realistic models will determine aspects of the dynamics of stellar envelopes that are robust and can be studied with our Boussinesq model. They may also suggest some intermediate (artificial) models where a baroclinic torque is applied in a spherically symmetric stellar model to study the dynamical aspects that are not sensitive to the true spheroidal shape.

Acknowledgements. I thank Matthieu Castro, Boris Dintrans, Bernard Pichon for providing various profiles of Brunt-Väisälä frequency out of stellar evolution codes and François Lignières, Sylvie Vauclair and Jean-Paul Zahn for many useful discussions. This work is part of the ESTER project aimed at modelling stars in two dimensions; it is supported by 
the Programme National de Physique Stellaire along with the Action Spécifique pour la Simulation Numérique en Astrophysique.

This work was started while the author was hosted at the Newton Institute during the programme "Magnetohydrodynamics of Stellar Interiors" which is gratefully acknowledged.

\section{References}

Braithwaite, J. 2006, A\&A, 449, 451

Brun, A. S., \& Toomre, J. 2002, ApJ, 570, 865

Busse, F. 1981, Geophys. Astrophys. Fluid Dyn., 17, 215

Busse, F. 1982, ApJ, 259, 759

Buzasi, D. L., Bruntt, H., Bedding, T. R., et al. 2005, ApJ, 619, 1072

Chandrasekhar, S. 1961, Hydrodynamic and hydromagnetic stability (Oxford: Clarendon Press)

de Souza, A. D., Kervella, P., Jankov, S., et al. 2005, A\&A, 442, 567

Garaud, P. 2002, MNRAS, 335, 707

Greenspan, H. P. 1969, The theory of rotating fluids (Cambridge University Press)

Knobloch, E. 1985, ApS\&S, 116, 149

Knobloch, E., \& Spruit, H. C. 1982, A\&A, 113, 261

Knobloch, E., \& Spruit, H. C. 1983, A\&A, 125, 59

Lignières, F., Rieutord, M., \& Reese, D. 2005, in Stellar pulsation and evolution, ed A. Walker, \& G. Bono, Mem. S. A. It., 76/4, 282
Maeder, A., \& Meynet, G. 2000, ARA\&A, 38, 143

Michaud, G., \& Proffitt, C. R. 1993, in Inside the Stars, IAU Coll., 137, ASP Conf. Ser., 40, 246

Morel, P. 1997, A\&AS, 124, 597

Peterson, D., Hummel, C., Pauls, T., et al. 2006, ApJ, 636, 1087

Rieutord, M. 1987, Geophys. Astrophys. Fluid Dyn., 39, 163

Rieutord, M. 1997, Une introduction à la dynamique des fluides (Paris: Masson)

Schneider, U. 1990, A\&A, 238, 142

Spiegel, E. A., \& Zahn, J.-P. 1970, Comments on Astrophysics and Space Physics, 2, 178

Spruit, H. C., \& Knobloch, E. 1984, A\&A, 132, 89

Stewartson, K. 1966, J. Fluid Mech., 26, 131

Tassoul, J.-L., \& Tassoul, M. 1982, ApJS, 49, 317

Tassoul, J.-L., \& Tassoul, M. 1983a, ApJ, 264, 298

Tassoul, J.-L., \& Tassoul, M. 1983b, ApJ, 271, 315

Tassoul, J.-L., \& Tassoul, M. 1984, ApJ, 279, 384

Tassoul, J.-L., \& Tassoul, M. 1986, Geophys. Astrophys. Fluid Dyn., 36, 303

Uryu, K., \& Eriguchi, Y. 1994, MNRAS, 269, 24

Uryu, K., \& Eriguchi, Y. 1995, MNRAS, 277, 1411

Zahn, J.-P. 1974, in Stellar Instability and Evolution, IAU Symp., 59, 185

Zahn, J.-P. 1992, A\&A, 265, 115

Zahn, J.-P. 1993, in Astrophysical fluid dynamics, Les Houches 1987, ed. J.-P. Zahn, \& J. Zinn-Justin (Elsevier), 561 